\title{
PSEUDOSCALAR NEUTRAL HIGGS BOSON PRODUCTION IN POLARIZED $\gamma$ e COLLISIONS
}

\author{
M. SAVCI * \\ Physics Department, Middle East Technical University \\ 06531 Ankara, Turkey
}

\begin{abstract}
We investigate the pseudoscalar neutral Higgs-boson $\mathcal{A}^{0}$ production in the polarized $\gamma e$ collisions with two different center of mass energies $\sqrt{s}=500 \mathrm{GeV}$ and $\sqrt{s}=1 \mathrm{TeV}$. The cross-section of the process $\gamma e \rightarrow e \mathcal{A}^{0}$ and the polarization asymmetry due the spin of the initial beams are calculated.
\end{abstract}

*e-mail: savci@rorqual.cc.metu.edu.tr 


\section{Introduction}

Despite that the Standard Model $(S M)$ describes very successfully all the experimental data within the range of energies available today, it has many unsolved problems, such as the problem of mass, $C P$ violation and the number of generations, etc., and the failure of the unification of electroweak and strong forces. For this reason one would expect that a more fundamental theory should exist, which describes the three forces within the context of a single group. Recent $L E P$ data shows that this indeed can be achieved in Supersymmetric Grand Unified Theories [1].

It is well known that the minimal supersymmetric extension of the $S M(M S S M)$ predicts the existence of many new particles (supersymmetric partners of the ordinary particles) and the Higgs sector of the theory contains two Higgs doublets in order to give mass to the up and down quarks [2]. The physical Higgs spectrum of the $M S S M$ is richer than the $S M$ and contains two $C P$-even and one $C P$-odd neutral Higgs bosons and a charged Higgs boson [3]. The $M S S M$ extension of the $S M$ leads to clear and distinct experimental signatures especially in the Higgs sector. The search for the Higgs sector of the MSSM constitutes one of the main research fields in the existing and future accelerators to which theoretical physicists focus their attention. A lot of theoretical work in the literature has been devoted to the production and decay channels of Higgs particles, in particular the pseudoscalar neutral Higgs-boson $\mathcal{A}^{0}$, in various reactions (see for example [4] and references therein). Here in this work, we investigate the production of the $\mathcal{A}^{0}$ in the polarized $\gamma e$ beams, namely in the process $\gamma e \rightarrow e \mathcal{A}^{0}$, at the center of mass energies $\sqrt{s}=500 \mathrm{GeV}$ and $\sqrt{s}=1 \mathrm{TeV}$. Note that production of the $\mathcal{A}^{0}$ without polarization in $\gamma e$ beams, is studied in [5]. However, the numerical results presented in that work, for the cross-sections of the the unpolarized beams, are over estimated and approximately 20 times larger than ours.

The paper is organized as follows. In sect. 2 we calculate the cross-section for $\mathcal{A}^{0}$ production. We present our numerical results and give a discussion about them in sect.3.

\section{Calculation of the Cross-Section $\gamma \mathbf{e} \rightarrow \mathbf{e} \mathcal{A}^{0}$ process}

The process $\gamma e \rightarrow e \mathcal{A}^{0}$ is described by two type of diagrams: box and triangle diagrams. From quite a number of diagrams, however, the main contributions arise from triangle ones with photon exchange. This is clear from the fact that, the photon pole diagrams give the 
dominant contributions since $t=0$ is in the physical region. Contributions of the triangle diagrams with $Z$-boson exchange and box diagrams give negligible contributions than that of photon exchange diagrams, since these diagrams are non-singular at $t=0$. Therefore these contributions are all disregarded in this present work. On the other hand since the $S U S Y$ extension of the $S M$ is considered, the charged chargino contributions are included.

The amplitude for the pseudoscalar $\mathcal{A}^{0}$ production in the $\gamma e \rightarrow e \mathcal{A}^{0}$ process can be written as

$$
\mathcal{M}=\frac{4 \alpha^{2}}{\sin \theta_{W} m_{W}} \bar{u}\left(p_{2}\right) \gamma_{\mu} u\left(p_{1}\right) \epsilon_{\rho} \frac{F(t)}{t}\left(p_{1}-p_{2}\right)_{\alpha} k_{1 \beta} \epsilon_{\mu \rho \alpha \beta} .
$$

Here $p_{2}$ and $p_{1}$ are the momenta of the final and initial electrons, respectively, $k_{1}$ and $\epsilon$ are the photon momentum and polarization, $t=-\left(p_{1}-p_{2}\right)^{2}$, and the loop factor $F(t)$ is given as,

$$
\begin{aligned}
F(t)= & {\left[-N_{C} Q_{t}^{2} m_{t}^{2} \cot (\beta) C_{0}\left(t, m_{\mathcal{A}^{0}}^{2}, m_{t}^{2}\right)-N_{C} Q_{b}^{2} m_{b}^{2} \tan (\beta) C_{0}\left(t, m_{\mathcal{A}^{0}}^{2}, m_{b}^{2}\right)\right.} \\
& -m_{\tau}^{2} \tan (\beta) C_{0}\left(t, m_{\mathcal{A}^{0}}^{2}, m_{\tau}^{2}\right)+2 m_{W} m_{1} g_{11} C_{0}\left(t, m_{\mathcal{A}^{0}}^{2}, m_{1}^{2}\right) \\
& \left.+2 m_{W} m_{2} g_{22} C_{0}\left(t, m_{\mathcal{A}^{0}}^{2}, m_{2}^{2}\right)\right]
\end{aligned}
$$

where, $N_{C}=3$ is the color factor, $Q_{t}=2 / 3$ and $Q_{b}=-1 / 3$ are the electric charge of the top and bottom quarks, $m_{1}$ and $m_{2}$ are the chargino masses and $\tan (\beta)$ is the ratio of the vacuum expectation values of the two Higgs Doublets (see for example [3]). The chargino coupling constants $g_{11}$ and $g_{22}$ depend on the elements of the $2 \times 2$ unitary matrices $U$ and $V$, that appear in diagonalization of the chargino mass matrix [2]:

$$
\chi=\left(\begin{array}{cc}
M & \sqrt{2} m_{W} \sin (\beta) \\
\sqrt{2} m_{W} \cos (\beta) & \mu
\end{array}\right),
$$

where, $M$ and $\mu$ are the symmetry breaking terms. Following [5], we also consider two different cases: $M \mu>m_{W}^{2} \sin (2 \beta)$ (case-1) and $M \mu<m_{W}^{2} \sin (2 \beta)$ (case-2), for which

$$
\begin{aligned}
& g_{11}=\frac{m_{W}}{m_{1}^{2}-m_{2}^{2}}\left[m_{2}+m_{1} \sin (2 \beta)\right], \\
& g_{22}=-\frac{m_{W}}{m_{1}^{2}-m_{2}^{2}}\left[m_{1}+m_{2} \sin (2 \beta)\right],
\end{aligned}
$$

and

$$
\begin{aligned}
& g_{11}=\frac{m_{W}}{m_{1}^{2}-m_{2}^{2}}\left[-m_{2}+m_{1} \sin (2 \beta)\right], \\
& g_{22}=-\frac{m_{W}}{m_{1}^{2}-m_{2}^{2}}\left[-m_{1}+m_{2} \sin (2 \beta)\right],
\end{aligned}
$$


respectively. In further analysis we will take $m_{1}>m_{2}$.

The scalar function $C_{0}$ entering in (2) is given as (since in the present case one of the external particles is a photon),

$$
C_{0}\left(t, m_{1}^{2}, m_{2}^{2}\right)=\frac{1}{t-m_{1}^{2}}\left[C\left(\frac{m_{1}^{2}}{m_{2}^{2}}\right)-C\left(\frac{t}{m_{2}^{2}}\right)\right]
$$

where,

$$
\begin{aligned}
C(x)= & \int_{0}^{1} \frac{d y}{y} \log [1-x y(1-y)-i \epsilon] \\
& =\left\{\begin{array}{cc}
2\left[\sinh ^{-1}\left(\sqrt{-\frac{x}{4}}\right)\right]^{2} & x \leq 0 \\
-2\left[\sin ^{-1}\left(\sqrt{\frac{x}{4}}\right)\right]^{2} & 0 \leq x \leq 4 \\
2\left[\cosh ^{-1}\left(\sqrt{\frac{x}{4}}\right)\right]^{2}-\frac{\pi^{2}}{2}+2 i \pi \cosh ^{-1}\left(\sqrt{\frac{x}{4}}\right) & x \geq 4 .
\end{array}\right.
\end{aligned}
$$

Using eq.(1) and performing summation over final electron spin and taking into account the polarization of the initial particle for the cross-section, we get

$$
\frac{d \sigma}{d(-t)}=\frac{1}{64 \pi s^{2}}|\mathcal{M}|^{2}
$$

where,

$$
|\mathcal{M}|^{2}=\frac{\alpha^{4}}{\sin ^{2} \theta_{W} m_{W}^{2}} \frac{|F(t)|^{2}}{(-t)}\left[s^{2}+u^{2}+\xi_{2} \lambda(s+u)^{2}\right] .
$$

Here we take $s=-\left(p_{1}+k_{1}\right)^{2}, u=-\left(p_{2}-k_{1}\right)^{2}$, and $\lambda$ and $\xi_{2}$ are the longitudinal polarization of the electron and circular polarization of the photon, respectively. From eq.(8) it follows that the expression for the differential cross-section contains the $\frac{1}{t}$ term and therefore in order to obtain the total cross-section for the subprocess, one must introduce a convenient cut-off parameter in the lower bound of the integration, namely, either the the mass of the electron or the cut-off parameter for the angle. By imposing the angular cut-off, i.e.,

$\eta=\sin ^{2}\left(\frac{\theta_{\min }}{2}\right) \simeq 10^{-5}$ the expression for the total cross-section takes the following form (see also [5]):

$$
\sigma_{\gamma e}(\hat{s})=\frac{\alpha^{4}}{64 \pi \sin ^{2} \theta_{W} m_{W}^{2}} \int_{\eta\left(\hat{s}-m_{\mathcal{A}^{0}}^{2}\right)}^{\left(\hat{s}-m_{\mathcal{A}^{0}}^{2}\right)} \frac{d y}{y}\left[\hat{s}^{2}+u^{2}+\xi_{2} \lambda(\hat{s}+u)^{2}\right]|F(y)|^{2},
$$

where, $\hat{s}=x s$ and $x$ is the momentum fraction of the electron carried out by the photon. Explicit calculations show that, the contribution of the $Z^{0}$ exchange diagram to the total cross-section is negligible than that of the photon exchange diagram. 
It is well known that the high energy $e^{+} e^{-}$colliders can be converted into high energy $\gamma e$ colliders with the help of the back-scattered laser beams [6]. So, taking into consideration the distribution function of the back-scattered photons, the total cross-section for the $\gamma e \rightarrow$ $e \mathcal{A}^{0}$ process can be calculated by

$$
\sigma=\int_{\frac{m_{A}^{2}}{s}}^{0.83} d x G_{\gamma}(x) \sigma(s x),
$$

where, $\sigma(s x)$ is given by eq.(10), the explicit form of the distribution function is [6]

$$
G_{\gamma}(x)=\frac{D_{1}}{D_{2}}
$$

where,

$$
\begin{aligned}
& D_{1}=1-x+\frac{1}{1-x}-\frac{4 x}{\xi(1-x)}+\frac{4 x^{2}}{\xi^{2}(1-x)^{2}}, \\
& D_{2}=\left(1-\frac{4}{\xi}-\frac{8}{\xi^{2}}\right) \log (1+\xi)+\frac{1}{2}+\frac{8}{\xi}-\frac{1}{2(1+\xi)^{2}} .
\end{aligned}
$$

In our numerical calculations we take $\xi=4.83$.

\section{Numerical Analysis}

We present our numerical results for the cross-sections for various values of $\tan (\beta)$ in a series of graphs. The dependence of the cross-section on $m_{\mathcal{A}^{0}}$ at different values of the circular polarization $\xi_{2}$ of the photon is presented in Fig.1 and Fig.2 for case-1 at $\sqrt{s}=$ $500 \mathrm{GeV}$ and $\sqrt{s}=1 \mathrm{TeV}$, respectively. A similar graphical analysis is illustrated for case-2 in Fig.3 and Fig.4. In the numerical calculations, for the largest chargino mass we take $m_{1}=250 \mathrm{GeV}$ and for $m_{2}$ we take the largest value consistent with the restriction $m_{1}-m_{2} \geq m_{W} \sqrt{2[1 \pm \sin (2 \beta)]}$, where upper and lower signs correspond to case-1 and case-2, respectively. Such restrictions are needed in order to ensure that $M$ and $\mu$ are real. The choice we made for $m_{1}$ and $m_{2}$ in this article, is within the range of the chargino masses that are found in the $S U S Y$ theories. Further, we checked for the contribution coming from $t, b, \tau$ and charginos separately. Our calculations show that, for larger values of $\tan (\beta)$ the contributions arising from $\tau$ lepton and charginos become important.

Our starting point is the analysis of case- 2 . For $\tan (\beta)=1$, the cross-section decreases with increasing values of $m_{\mathcal{A}^{0}}$ and around $m_{\mathcal{A}^{0}} \simeq m_{t}$, it reaches a deep minimum value, which from that point on it starts increasing and attains its maximum value at $m_{\mathcal{A}^{0}} \simeq 2 m_{t}$, 
and then decreases smoothly again. For larger values of $\tan (\beta)$ (i.e., for $\tan (\beta)=5,20$ and 50 ), the behavior of the cross-section is absolutely different and it gets its first maximum value around $m_{\mathcal{A}^{0}} \simeq m_{t}$, contrary to the previous case. The second maximum point is again around $m_{\mathcal{A}^{0}} \simeq 2 m_{t}$. Note that, at $\sqrt{s}=1 \mathrm{TeV}$, around the point $m_{\mathcal{A}^{0}} \simeq 500 \mathrm{GeV}$, a new maximum value for the cross-section shows itself, which is totally absent in the $\sqrt{s}=500 \mathrm{GeV}$ case. It is interesting to observe that, the locations of the maximum values of the cross-section are all independent of $\tan (\beta)$. Similar situation holds for all other values $\xi_{2}$ of the circular polarization of the photon. The different behavior of the cross-section for the small and large values of $\tan (\beta)$ is due to the increase in the contributions arising from the $\tau$-lepton and charginos.

Similar arguments hold for case-1 as well, but with the following differences: For almost all values of $\tan (\beta)$ the cross-section has its maximum value located around the same point $m_{\mathcal{A}^{0}} \simeq m_{t}$, while for $\tan (\beta)=20$ and $\tan (\beta)=50$ it has a minimum around the point $m_{\mathcal{A}^{0}} \simeq 150 \mathrm{GeV}$.

For all cases the maximum value of the cross-section is about $\sim 0.1 \mathrm{fb}$. If we assume that the integral luminosity at $\sqrt{s}=500 \mathrm{GeV}$ is $10 \mathrm{fb}^{-1}$ and at $\sqrt{\mathrm{s}}=1 \mathrm{TeV}$ is $60 \mathrm{fb}^{-1}$ [6], we have at most 2 events for $\sqrt{s}=500 \mathrm{GeV}$ and 12 events for $\sqrt{s}=1 \mathrm{TeV}$.

From the analysis of all graphs, we can deduce the following result: For the detection of $\mathcal{A}^{0}$, the $\tan (\beta)=1$ case is more preferable. At $\sqrt{s}=500 \mathrm{GeV}$, when $g_{11}=-g_{22}$ $\left(g_{11}=g_{22}\right)$, it seems that it is possible to detect $\mathcal{A}^{0}$ with a mass around $m_{\mathcal{A}^{0}} \simeq m_{t}$ $\left(m_{\mathcal{A}^{0}} \simeq 2 m_{t}\right)$. Similar situation is present for $\sqrt{s}=1 \mathrm{TeV}$, but with one important difference. For the case $g_{11}=-g_{22}$ at $\tan (\beta)=1$, it is possible to detect $\mathcal{A}^{0}$ with a mass around $m_{\mathcal{A}^{0}} \simeq m_{t}$ and $m_{\mathcal{A}^{0}} \simeq 2 m_{t}$. Note that for this value of the $\tan (\beta)$, the cross-section for the case $g_{11}=-g_{22}$, is larger than that of $g_{11}=g_{22}$.

Few words about the possibility of detecting $\mathcal{A}^{0}$ in experiments should be mentioned. In order to detect $\mathcal{A}^{0}$ in real experiments, we must take the decay products of $\mathcal{A}^{0}$ into account and separate the background processes from the signal of the $\mathcal{A}^{0}$ decay with the same final state particles. The main decay channels of $\mathcal{A}^{0}$ are $\llbracket$, $\mathbb{7}: \mathcal{A}^{0} \rightarrow b \bar{b}, c \bar{c}, t \bar{t}, \tau^{+} \tau^{-}$, $W^{ \pm} W^{\mp}, Z h, Z H, g g, \gamma \gamma$ and $Z \gamma$. If $m_{\mathcal{A}^{0}} \leq 2 m_{t}$, in the minimal supersymmetric models the dominant decay mode of $\mathcal{A}^{0}$ is $\mathcal{A}^{0} \rightarrow b \bar{b}$ (for more detail see $\llbracket$ ). This decay mode is dominant even above the top quark threshold up to $\tan \beta \simeq 30$ [8. In this case we can also neglect the chargino contributions since they are smaller than the one arising from $b \bar{b}$ mode (see [4]). Therefore, in our case the main process which is responsible for detecting 
$\mathcal{A}^{0}$ is $\gamma e \rightarrow e \mathcal{A}^{0} \rightarrow e b \bar{b}$ with a branching ratio of $\operatorname{Br}\left(\mathcal{A}^{0} \rightarrow b \bar{b}\right) \simeq 0.95$ [4]. It is clear that in this case the main background process is the direct $b \bar{b}$ production in $\gamma e \rightarrow e b \bar{b}$ (see [5]). A comparison of our results on cross-section and the background process $\gamma e \rightarrow e b \bar{b}$ [5] leads to the conclusion that at $\sqrt{s}=500 \mathrm{GeV}$ the background process dominates for all values of $\tan \beta$. On the other hand at $\sqrt{s}=1 T e V$, and $\tan \beta=1$ the $b \bar{b}$ signal from $\mathcal{A}^{0}$ can be detected with a mass $m_{\mathcal{A}^{0}} \simeq 2 m_{t}$.

Finally we would like to discuss the following question: Can we deduce any extra information about the mass of $\mathcal{A}^{0}$, if we investigate the polarization asymmetry due to the longitudinal and circular polarization of the initial electron and photon, respectively? In order to answer this question, we presented in Fig.(5) the behavior of polarization asymmetry versus $\sqrt{s}$ at different values of $m_{\mathcal{A}^{0}}$. We clearly observe that when $m_{\mathcal{A}^{0}} \simeq m_{t}$ polarization asymmetry approaches to zero around $\sqrt{s} \simeq 1.7 \mathrm{TeV}$, and this value of energy is far from the maximum available energy range of the present colliders. Moreover, it follows from this figure that, the dependence of polarization asymmetry on $\sqrt{s}$ at different values of $m_{\mathcal{A}^{0}}$, is practically the same. Therefore, in our opinion, the polarization asymmetry is not a convenient tool for deducing extra information about the mass of $\mathcal{A}^{0}$.

Our final conclusion is that, in search of the pseudo-scalar $\mathcal{A}^{0}$ in $\gamma e$ collider, with the preferred choice of $\tan (\beta)=1$, it is possible to detect the pseudoscalar $\mathcal{A}^{0}$ with a mass of $m_{\mathcal{A}^{0}} \simeq 2 m_{t}$. 


\section{Figure Captions}

Fig.1. The dependence of the cross-section of $\mathcal{A}^{0}$ production in $\gamma e \rightarrow e \mathcal{A}^{0}$ on the mass $m_{\mathcal{A}^{0}}$ for various values $\tan \beta$ and circular polarization $\xi_{2}$ of the photon at a fixed value of the longitudinal polarization of the initial electron $(\lambda=0.9)$, for the case $M \mu>m_{W}^{2} \sin (2 \beta)$ at the center of mass energy $\sqrt{s}=500 \mathrm{GeV}$. The mass of the charginos are given in the text.

Fig.2. The same as in Fig.1, but for $\sqrt{s}=1 \mathrm{TeV}$.

Fig.3. The same as in Fig.1 but for the case $M \mu<m_{W}^{2} \sin (2 \beta)$.

Fig.4. The same as in Fig.3 but for $\sqrt{s}=1 \mathrm{TeV}$.

Fig.5. Polarization asymmetry $\mathcal{A}=\frac{\sigma\left(\xi_{2}=+1\right)-\sigma\left(\xi_{2}=-1\right)}{\sigma\left(\xi_{2}=+1\right)+\sigma\left(\xi_{2}=-1\right)}$ versus $\sqrt{s}$ for the case $M \mu<m_{W}^{2} \sin (2 \beta)$, for different values of $m_{\mathcal{A}^{0}}$ and at $\tan \beta=1, \lambda=0.9$. For all other choices of the parameters $\tan \beta, \xi_{2}$ and the case $M \mu>m_{W}^{2} \sin (2 \beta)$, the graphical analysis yields almost identical figures. 


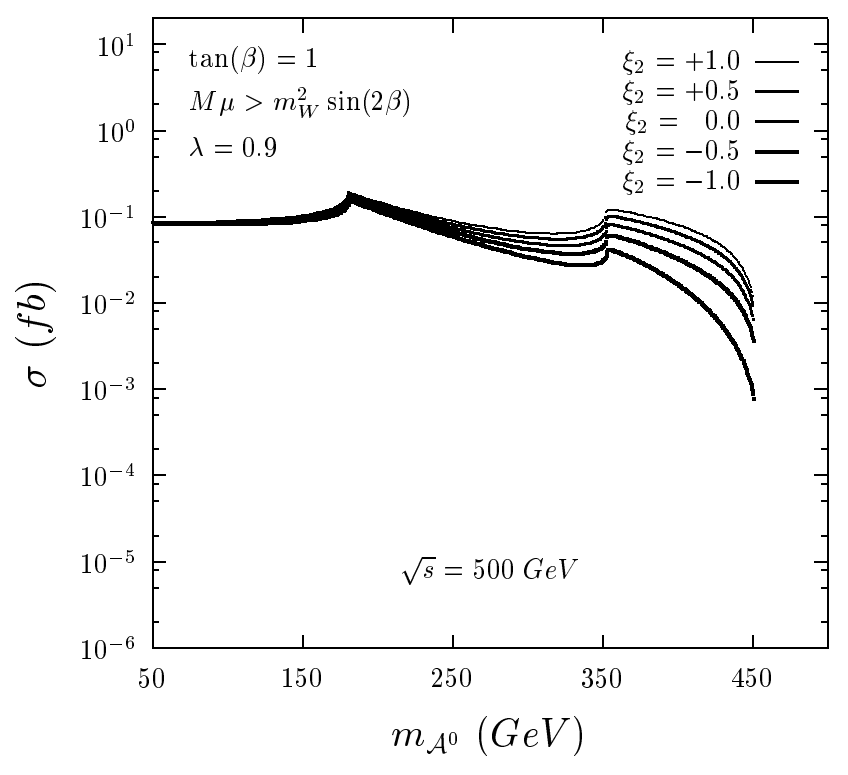

Fig.1.a

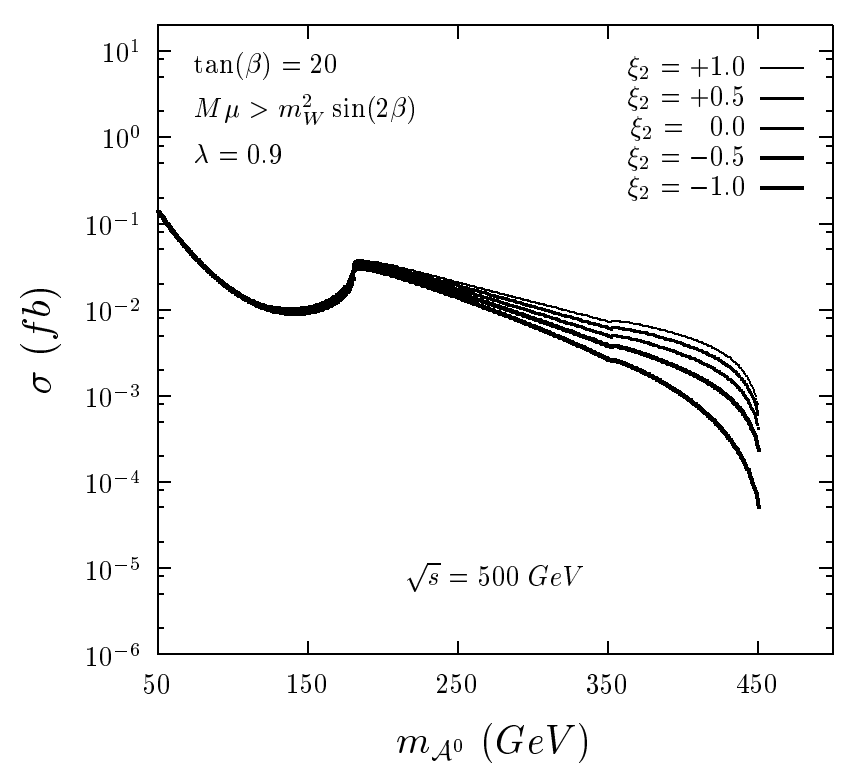

Fig.1.c

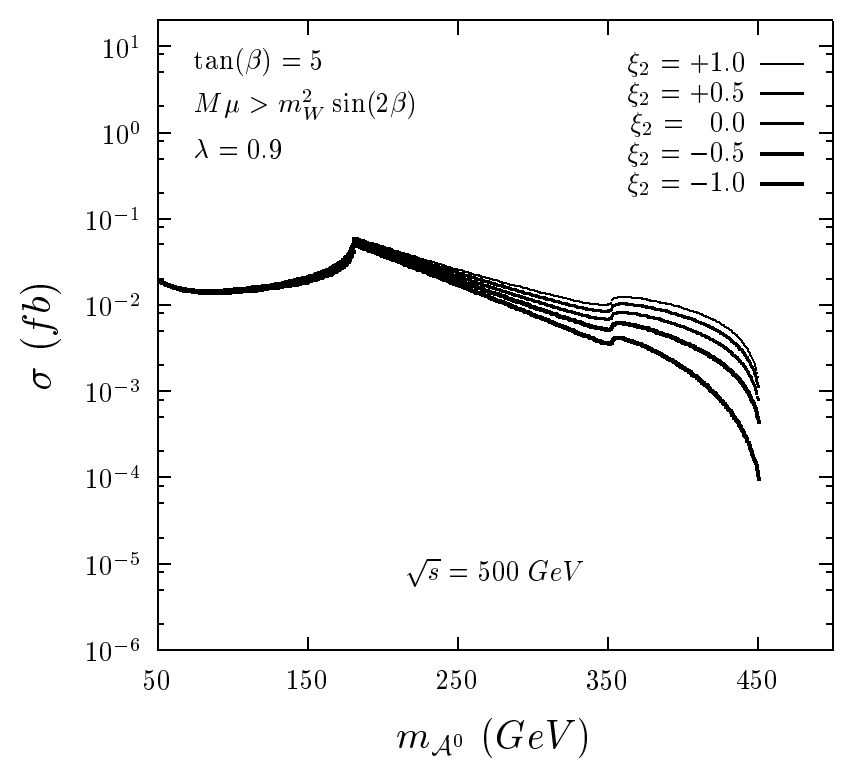

Fig.1.b

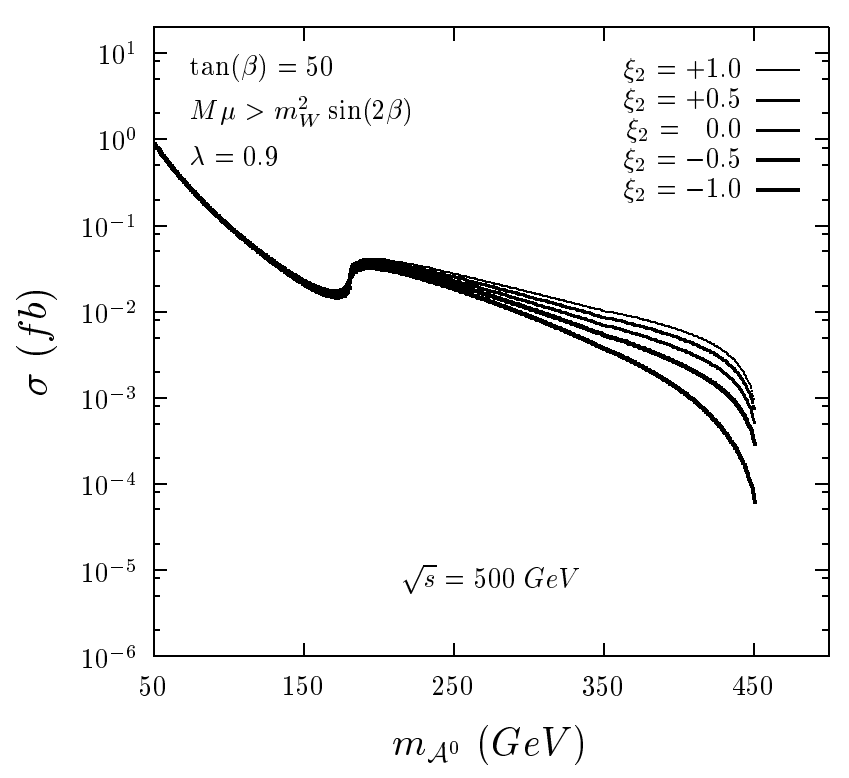

Fig.1.d

Figure 1: Case-1, for $\sqrt{s}=500 \mathrm{GeV}$. 


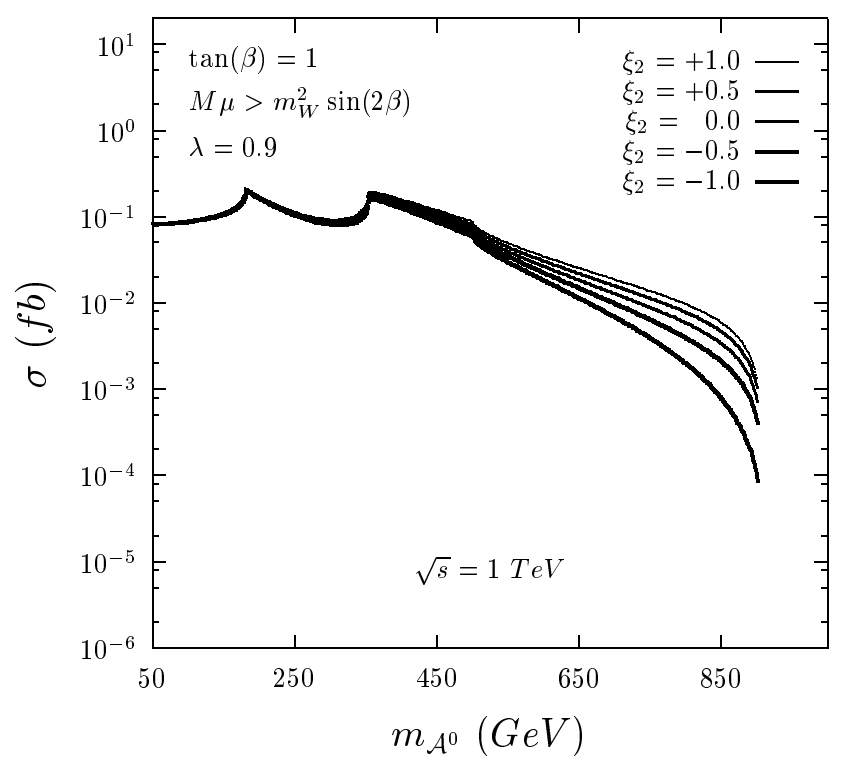

Fig.2.a

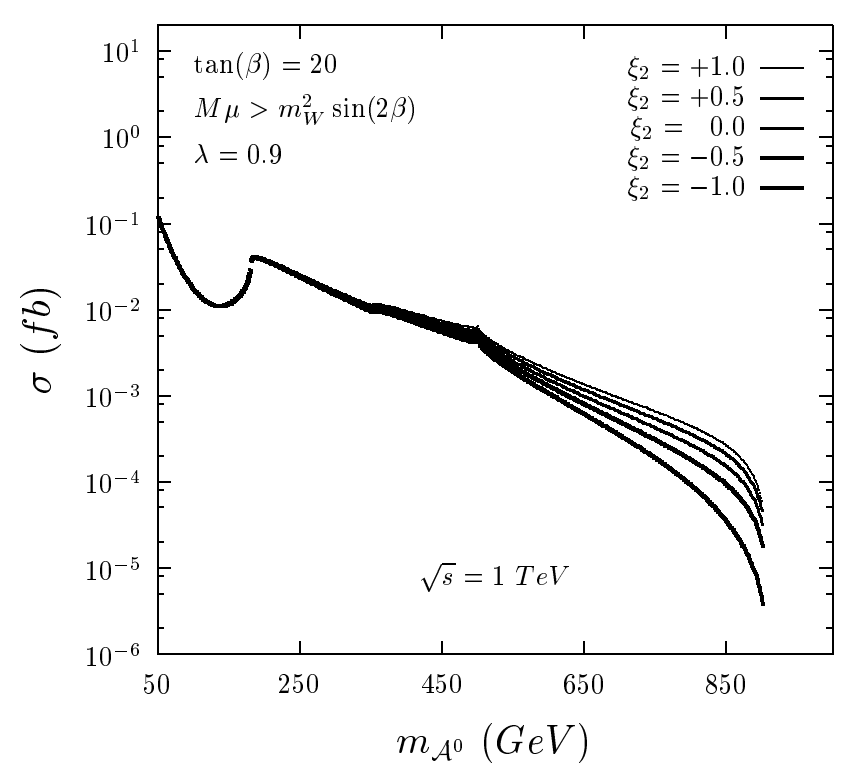

Fig.2.c

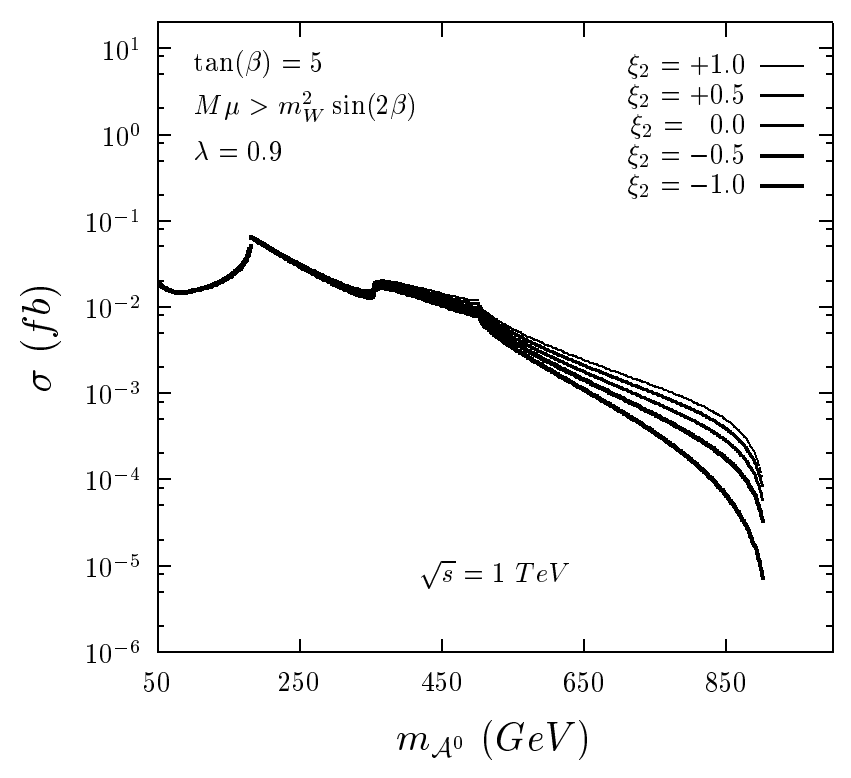

Fig.2.b

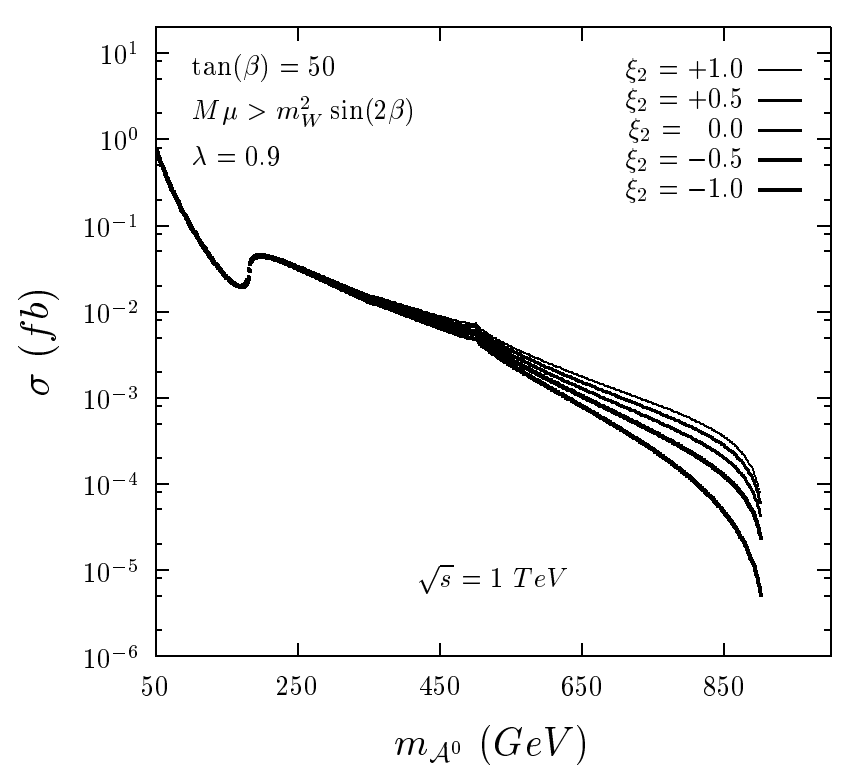

Fig.2.d

Figure 2: Case-1, for $\sqrt{s}=1 \mathrm{TeV}$. 


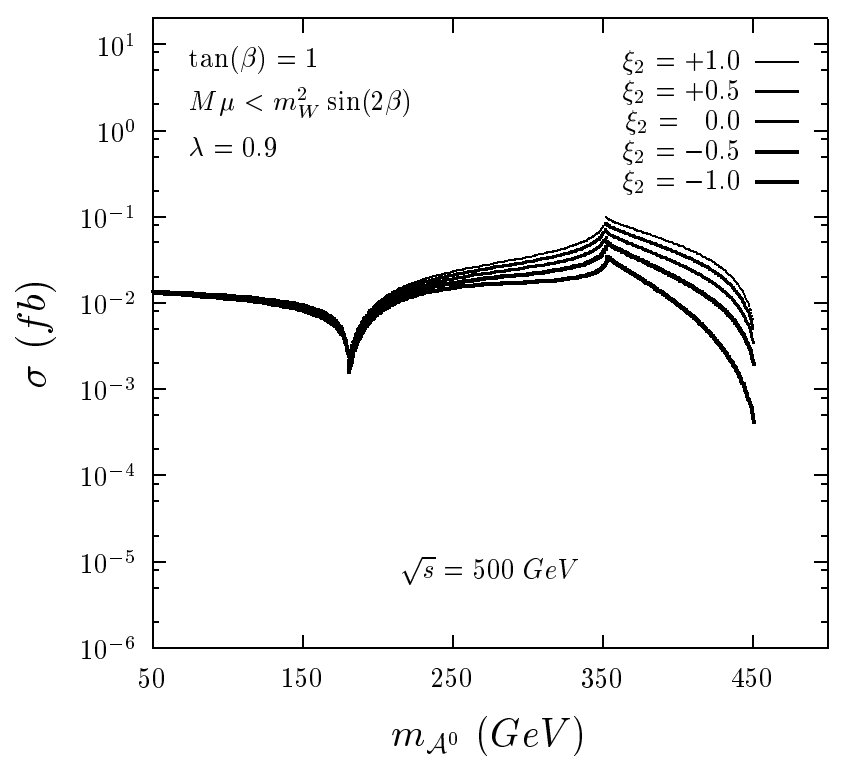

Fig.3.a

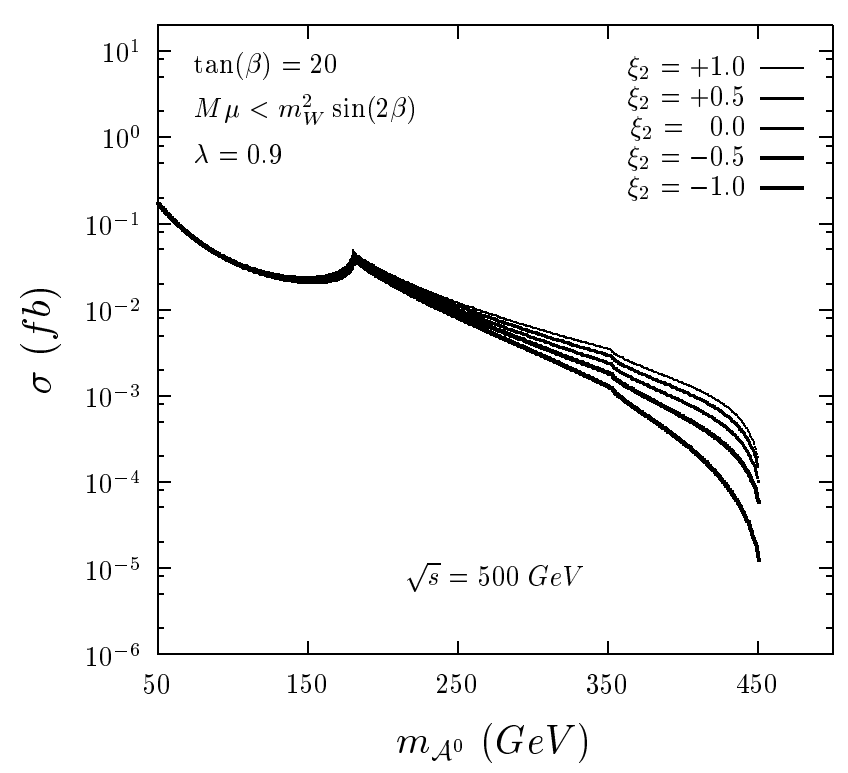

Fig.3.c

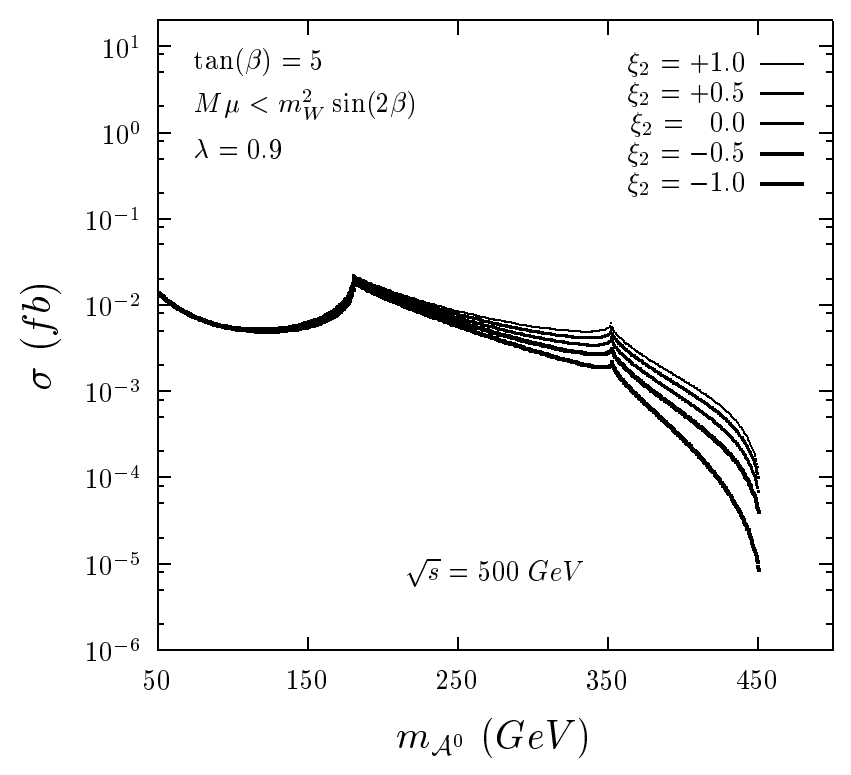

Fig.3.b

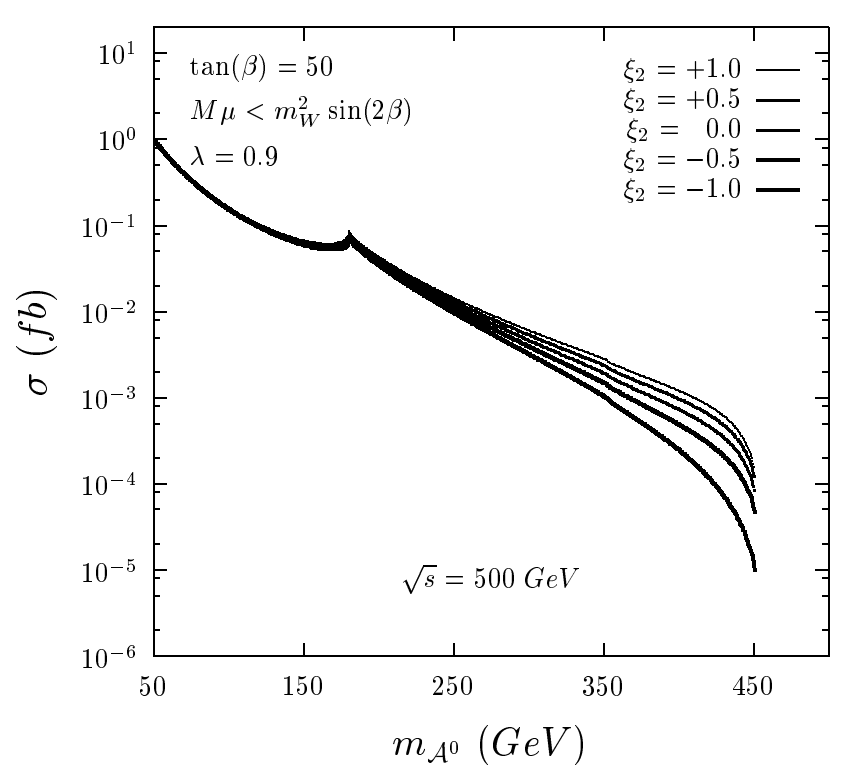

Fig.3.d

Figure 3: Case-2, for $\sqrt{s}=500 \mathrm{GeV}$. 


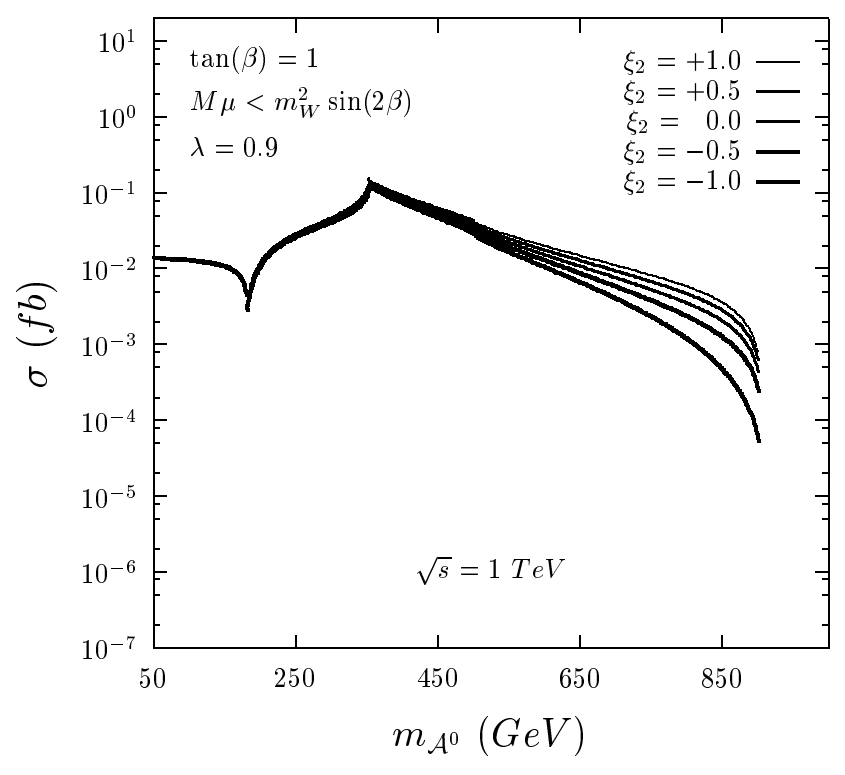

Fig.4.a

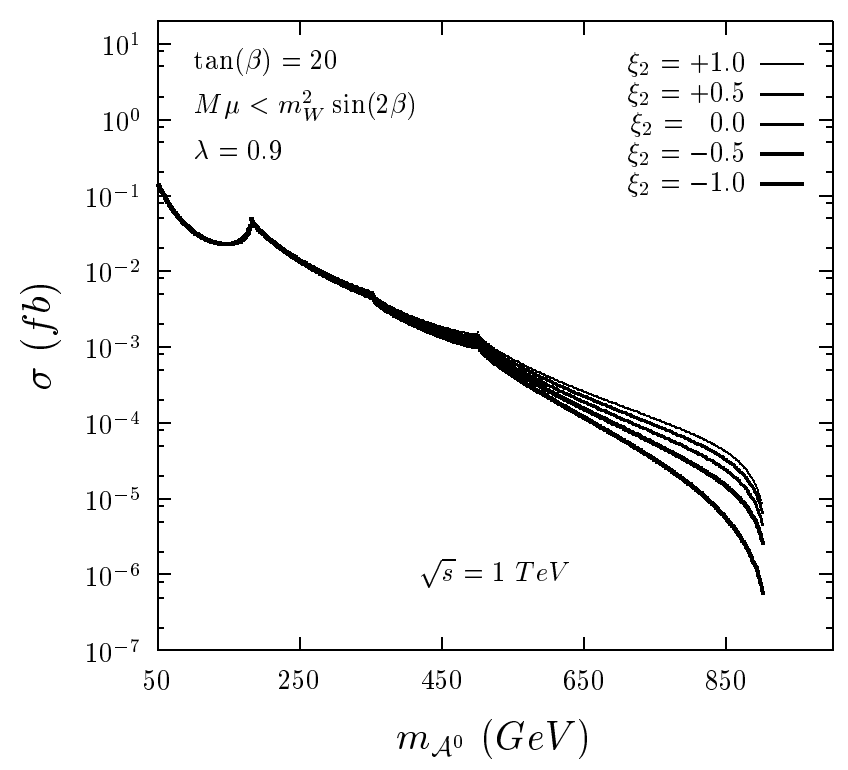

Fig.4.c

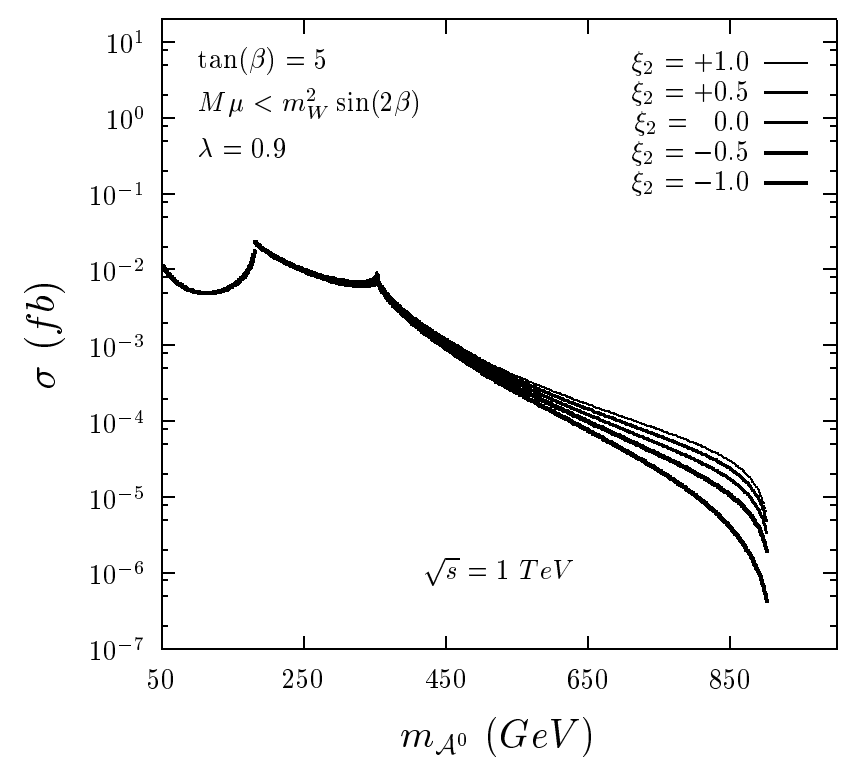

Fig.4.b

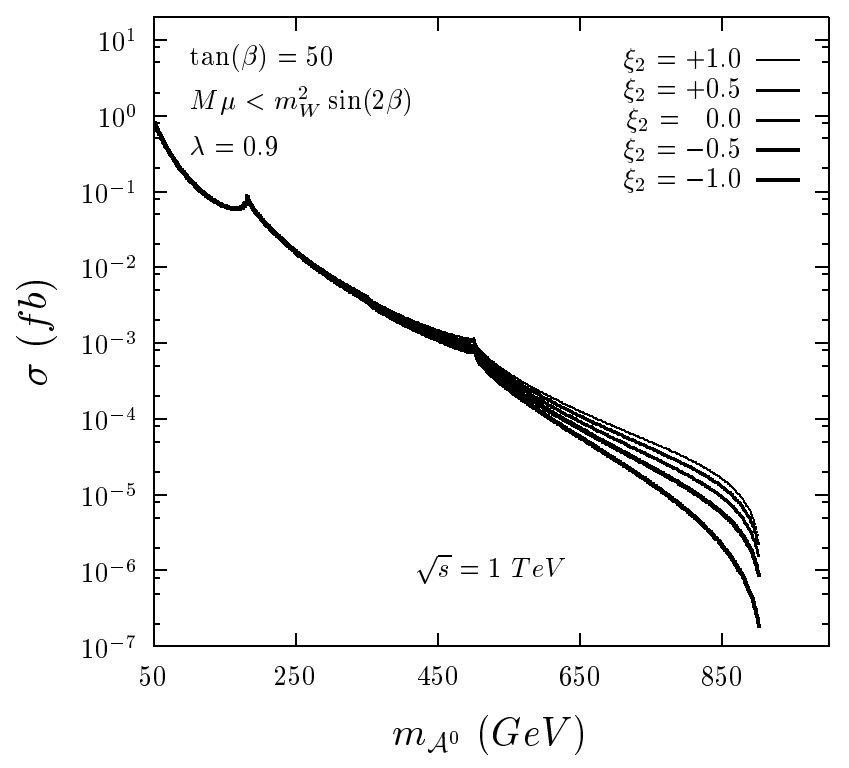

Fig.4.d

Figure 4: Case-2, for $\sqrt{s}=1 \mathrm{TeV}$. 


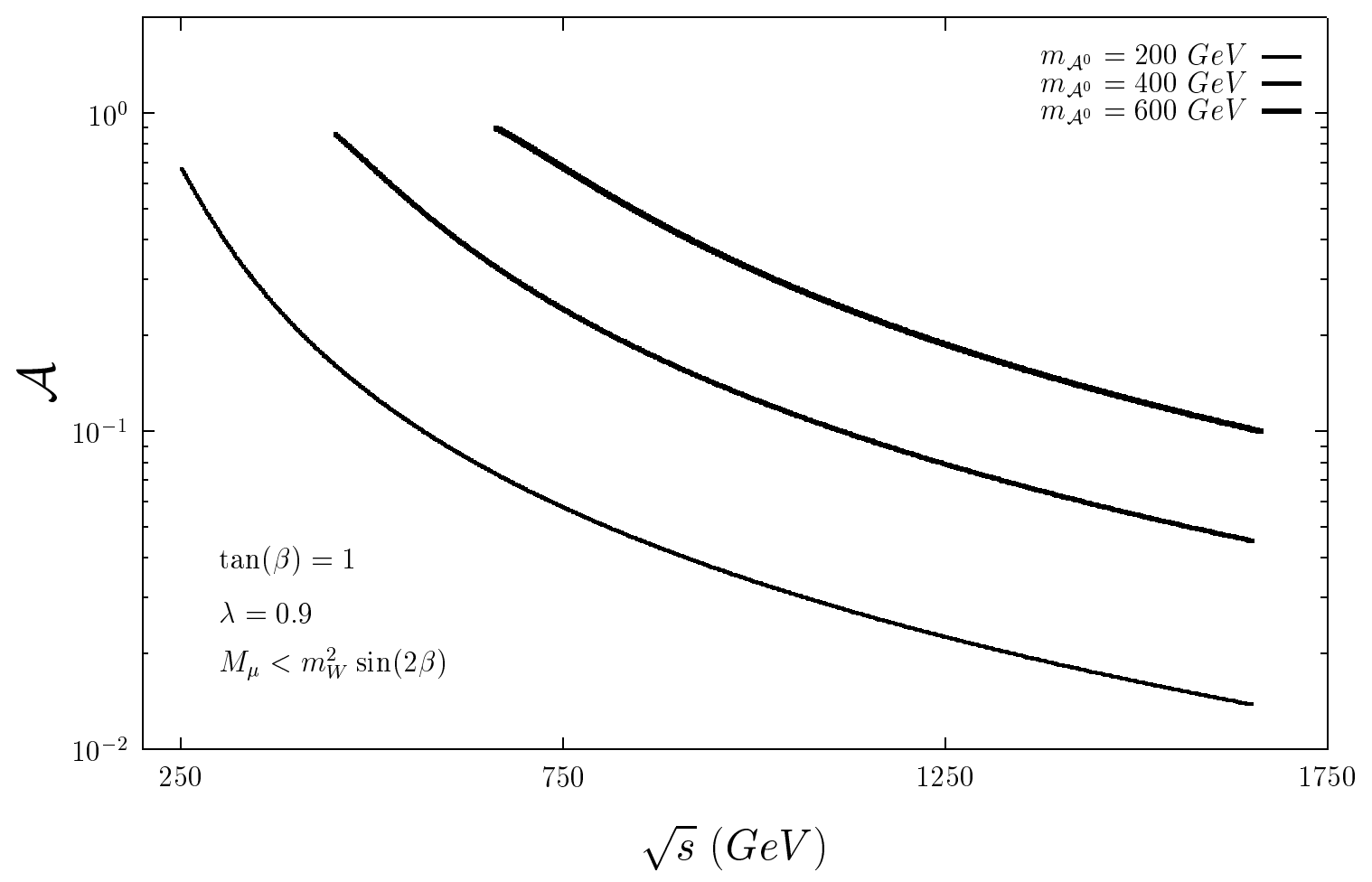

Figure 5: Polarization asymmetry. 


\section{Acknowledgements}

I am deeply indebted to Prof. Dr. T. M. Aliev, for invaluable contributions and clarifying discussions throughout the whole course of the work. 


\section{References}

[1] J. Ellis, S. Kelley and D. V. Nanopoulos, Phys. Lett. B260 (1991) 131.

U. Amaldi, W. de Boer and H. Fürstenau, Phys. Lett. B260 (1991) 447.

P. Langacker and M. Luo, Phys. Rev. D44 (1991) 817.

[2] H. E. Haber and G. L. Kane, Phys. Rep. C117 (1985) 75.

[3] J. F. Gunion, H. E. Haber, G. L. Kane and S. Dawson, The Higgs Hunter's Guide, Addison-Wesley, (1990).

[4] Proc. "Physics at LEP2" CERN 96-01 (1996),

Eds. G. Altarelli, T. Sjöstrand and F. Zwirner.

[5] Duane A. Dicus and Wayne W. Repko, Phys. Rev. D53 (1996) 3616.

[6] For a review see, V. Telnov, Nucl. Instrum. Methods A294 (1990) 72.

[7] D. Kominis, Nucl. Phys. B427 (1994) 575.

[8] V. Barger, K. Chung, R. J. N. Phillips and A. L. Strange, Phys. Rev. D46 (1992) 4914. 